\title{
LE COEFFICIENT DE RENDEMIENT EN FROMAGERIE NÉCESSITE D'UNE DÉFINITION NOUVELLE
}

\author{
par \\ JEAN PIEN \\ Ingénieur-Chimiste, Docteur ès Sciences \\ Directeur des Laboratoires de la Laiterie des Fermiers Réunis
}

Dans une étude récente sur les bases techniques de la législation et de la fabrication des fromages (1) nous avons souligné l'importance de la détermination du "coefficient de rendẹment" (que nous avons appelé coefficient $\mathbf{K}$ ) c'est-à-dire du poids d'extrait sec non gras que peut fournir, sous forme de fromage, un litre de lait de mélange mis en fabrication.

Ce coefficient est à la base de Ja plupart des calculs relatifs à la composition des fromages et au rendement des fabrications. La "valeur fromagère" du lait lui est donc liée en grande partie. Il n'est pas douteux que lorsque l'on aura des précisions plus grandes sur cette "donnée " importante, elle pourra et devra entrer en ligne de compte dans le calcul du prix d'achat du lait en fromagerie. Tous les efforts des techniciens de l'industrie fromagerie doivent done tendre vers une étude approfondie en vue d'une meilleure connaissance de ce "coefficient de rendement".

De très nombreux problèmes, en effet, se posent à son sujet. Les plus importants concernent les causes et conditions de ses variations. Il y en a d'autres qui paraissent encore plus urgents à résoudre ear, selon nous, ils mettent en cause la définition même du coefficient.

La présente note n'a d'autre objet que d'attirer l'attention sur ces variations et de poser le problème d'une définition nouvelle du coefficient ou, plus exactement, de proposer un coefficient différent répondant à une définition plus rigoureuse.

\section{*** \\ LES FACTEURS DE VARIATION DU COEFFICIENT DE RENDEMENT}

Les causes qui affectent la valeur du coefficient de rendement sont nombreuses. On peut, semble-t-il, les classer en deux groupes: celles qui dépendent du lait et celles qui sont inhérentes aux fromages eux-mêmes.

(1) Le Lait, janvier-mars 1945, page 1. 


\section{Causes de variation dues au lait}

Nous ne devons pas perdre de vue que l'extrait sec non gras qui passe dans le fromage au cours de la fabrication dépend avant tout (et entre autres nombreuses choses) de l'extrait sec dégraissé du lait. On peut donc admettre, a priori, que les causes qui font varier l'extrait dégraissé du lait influenceront dans le même sens le coefficient de rendement en fromagerie.

\section{Variation en fonction des régions et des saisons.}

Les variations de l'extrait dégraissé du lait suivant les régions sont surtout fonction des races locales et de la nature de l'alimentation. En fonction du temps, cet extrait évolue d'une manière rythmique au cours du cycle d'une année. Il passe périodiquement par des minima et des maxima saisonniers que l'on retrouve avec une certaine régularité dans les laits de grand mélange. De sorte qu'à la même saison, dans une même région, on constate à peu de choses près les mêmes extraits secs dégraissés, pour autant, répétonsle, que les autres facteurs (race, alimentation...) n'aient pas changé - ce qui n'est malheureusement pas le cas actuellement...

Mais, si les variations de l'extrait dégraissé du lait en fonction du temps et des lieux peuvent, dans l'ensemble, être assez bien connues en période normale, nous manquons jusqu'ici de documents sur les variations correspondantes du coefficient de rendement en fromagerie.

\section{$2^{\circ}$ Variation en fonction de la richesse en matière grasse du lait.}

Il semble acquis que la matière grasse du lait facilite la rétention de l'extrait sec dans le fromage. Toutes choses égales d'ailleurs, un lait plus riche donne un coefficient de rendement (extrait non gras) plus élevé.

Mais eette notion demande à être confirmée, car nous devons reconnaître que, dans certains cas, et sans qu'on ait pu jusqu'ici en découvrir la raison, le résultat inverse a été constaté. Il faut d'ailleurs noter sincèrement que les expériences de détermination du coefficient de rendement sont extrêmement difficiles à réussir et certains résultats aberrants sont incontestablement dus à des imperfections de la technique expérimentale qu'il faudra préciser et améliorer sans cesse.

\section{Variations dues au fromage lui-même}

Dès que la lait de mélange est emprésuré, de nouvelles causes de variations interviennent qui se superposent à celles que nous venons de rappeler brièvement. Notons entre autres : 


\section{Variations en fonction du mode de fabrication.}

Tous les facteurs qui influencent l'égouttage c'est-à-dire qui conditionnent la sortie du sérum et même, dans une certaine mesure, les pertes de caséine dans ce sérum, vont jouer un rôle dans l'élaboration de l'extrait sec du fromage : acidité du lait avant l'emprésurage, conditions de l'égouttage proprement dit (spontané ou provoqué par rompage suivi de pressage), etc...

Il va de soi qu'un fromage dans lequel on laissera volontairement davantage de sérum fournira, par litre de lait mis en œuvre, un extrait sec plus important. C'est la raison pour laquelle le coefficient de rendement, tel qu'il a été défini, est plus élevé dans les pâtes molles que dans les pâtes pressées.

Pour fixer les idées, nous emprunterons à l'excellent ouvrage de M. A. M. Gúfradlt (L'Industrie laitière. Organisation et Gestion des Entreprises. C. E. P., éditeur, 31, rue de Rome, à Paris) les chiffres suivants qui illustrent ce que nous venons de dire et tendent d'autre part, à apporter la confirmation du fait suivant lequel la matière grasse favorise la rétention de l'extrait sec.

\begin{tabular}{|c|c|c|}
\hline \multirow{2}{*}{$\begin{array}{l}\text { Richesse du fromage } \\
\text { en matière grasse } \% \text { de sec }\end{array}$} & \multicolumn{2}{|c|}{ Coefficient de rendement (1) } \\
\hline & Pâtes molles & Pâtes pressées \\
\hline$\ldots \ldots \ldots$ & 32 à 34 & 30 à 31,5 (2) \\
\hline $40 \% \quad \ldots \ldots \ldots \ldots \ldots \ldots$ & 31 à 33 & 29 à $30,5(2)$ \\
\hline $30 \% \ldots \ldots \ldots \ldots \ldots \ldots$ & 30,5 à 32,5 & 28,5 à 30 \\
\hline $20 \% \ldots \ldots \ldots \ldots \ldots \ldots$ & 30 à 32 & 28 à 29,5 \\
\hline
\end{tabular}

Ces chiffres, dans l'esprit même de l'auteur, n'ont encore qu'une valeur relative. Il faut les regarder, pour le moment, comme des ordres de grandeur. Ils devront être étudiés, vérifiés, précisés au cours de nombreuses expériences.

\section{$2^{\circ}$ Variations en fonction de l'âge du fromage.}

Cette notion est d'une importance capitale.

Nous avons montré (3) qu'au cours de leur maturation et de leur conservation, les fromages - notamment les pâtes molles -

(1) Ce coefficient reçoit, dans. l'ouvrage de M. Gú́raút, le nom de coeffícient G parce qu'il représente le facteur n 7 d'une série d'éléments que cet auteur utilise pour l'étude de rendement théorique. Cette expression possède évidemment la même signification que celle que nous avons attribuée au terme « coefficient $K$.

(2) Le cas du gruyère étant mis à part (coefficients nettement plus faibles encore).

(3) Le Lait, décembre 1937, p. 1040. 
subissent une perte de matière sèche non grasse (formation de substances volatiles) qui augmente le taux de matière grasse pour cent de matière sèche. Il est évident que, pour la même raison, le taux de matière sèche non grasse du fromage rapporté à un litre de lait subira une diminution progressive au cours de l'affinage.

A titre purement documentaire voici quelques exemples concernant des camemberts analysés 10 jours et 20 jours après la fabrication :

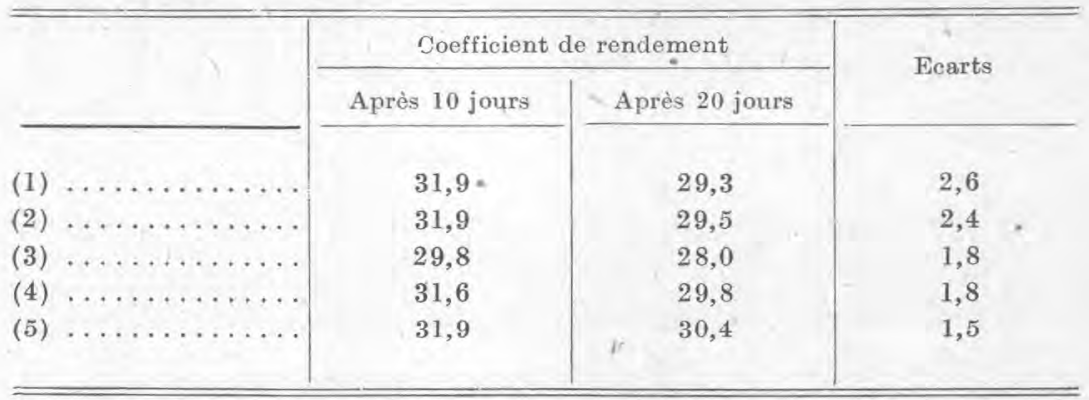

Comme on le voit, les écarts spont relativement importants et variables suivant, bien entendu, les conditions de l'affinage. Des écarts plus grands ont été constatés entre les premiers jours qui suivent la fabrication et la fin de l'affinage.

Cette donnée importante qui demande, elle aussi, à être précisée, nous montre à coup sûr la nécessité de définir le coefficient de rendement à l'aide d'analyses effectuées le plus tôt possible, dès que l'égouttage est terminé ou au plus tard dans les 24 ou 48 heures qui suivent.

\section{$3^{\circ}$ Variations individuelles.}

Lorsqu'on effectue l'analyse d'un fromage isolé issu d'une fabrication industrielle, on n'obtient en général, pour le coefficient de rendement, que des résultats approchés, souvent inexacts, car un fromage isolé ne représente jamais la moyenne d'un lot. Il faut absolument, comme le recommande d'ailleurs M. GUÉRAULT (loc. cit.) effectuer l'analyse sur la pâte obtenue en malaxant ensemble un certain nombre de fromages afin de faire disparaître les causes de variations individuelles.

Un autre moyen d'échapper à cette difficulté consiste à réaliser une fabrication de laboratoire sur un très petit volume de lait (par exemple préparer deux camemberts avec 4 litres de lait) en se plaçant autant que possible dans les conditions de la fabrication. A priori, cette méthode peut paraître eritiquable. En fait, elle a le grand mérite de permettre des mesures de volumes et des pesées 
très précises ; elle autorise en outre la mise en œuvre de la totalité de la "fabrication " en vue de l'analyse. Pour le but poursuivi ici (qui ne vise pas à réaliser l'affinage) cette méthode, susceptible d'ailleurs d'être perfectionnée à l'extrême, semble devoir donner d'excellents résultats. Il y aura intérêt à la mettre en parallèle avec la méthode semi-industrielle dont l'application est, pratiquement, si difficile.

Cette technique simplifiée aurait enfin l'avantage de permettre de nombreux essais en parallèle, ce qui rendrait plus facile l'étude des nombreux facteurs de variation du coefficient de rendement, dont nous venons seulement d'énumérer les principaux.

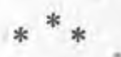

\section{L'AMÉLIORATION DE LA DEFINITION DU COEFFICIENT DE RENDEMENT}

La définition donnée jusqu'ici du coefficient de rendement comporte au moins deux erreurs de principe dont il convient, à notre avis, de se débarrasser.

On dit, en effet, que ce coefficient se rapporte à un litre de lait de mélange (dont la richesse en matière grasse n'est pas définie). D'autre part, l'analyse du fromage est effectuée nécessairement sur le fromage salé et l'on compte, comme extrait sec apporté par le lait, le sel introduit dans la fabrication, lequel.d'ailleurs ne reste pas intégralement dans le fromage.

Voyons quelle peut être l'influence de ces deux erreurs sur la valeur du coefficient.

\section{Erreur due à la richesse en matière grasse du lait de mélange}

L'extrait sec dégraissé du lait n'a pas de signification très précise si on le rapporte au lait entier. En effet : prenons un même lait entier et écrémons-le à divers taux; la partie non grasse de ce lait possède une composition constante (le litre de lait écrémé contenu dans ce lait entier a un extrait sec défini). En revanche, cet extrait dégraissé, ramené au litre de lait entier, varie suivant le taux de matière grasse et augmente quand la matière grasse diminue. C'est pourquoi, depuis longtemps, on a préconisé l'utilisation de l'extrait dégraissé "rectifié " (PORCHER), c'est-à-dire ramené à un litre de lait écrémé.

De même, pour un certain lait entier, l'extrait non gras susceptible de passer dans le fromage est bien défini pour un litre de lait écrémé contenu dans ce lait entier. Si, dans ce même lait, nous 
venons à faire varier le taux de matière grasse, la quantitè de lait écrémé par litre de lait entier va, elle aussi, varier et le coefficient de rendement, rapporté au lait de mélange, ne sera plus la constante dont nous avons besoin.

De toute évidence, il faut que ce coefficient soit un caractère fondamental du lait, susceptible de définir sa qualité fromagère ; il doit donc rester indépendant, par exemple, des opérations d'écrémage partiel qui précèdent les fabrications.

Il n'y a évidemment qu'un moyen de tourner cette difficulté. C'est de remplacer le coefficient utilisé jusqu'ici, par un coefficient "rectifié" qu'on pourrait définir ainsi : "Poids de matière sèche non grasse que peut théoriquement fournir, sous forme de fromage, un litre de lait totalement écrémé appartenant au lait entier considéré. "

Il ne s'agit pas pratiquement de savoir ce que donnerait réellement un litre de ce lait après écrémage total. Il s'agit de prendre le coefficient ancien et de le ramener par le calcul á un litre de lait supposé écrémé.

Quelle est l'incidence de cette opération ?

Appelons K le coefficient classique rapporté au lait entier. (1) $\mathrm{R}$ la richesse au litre du lait entier ;

" $\quad K_{1}$ le nouveau coefficient (rapporté au litre de lait écrémě).

Le volume des $R$ gr. de matière grasse est égal à $\frac{R}{0,915}\left(\mathrm{~cm}^{3}\right)$.

Le volume du lait écrémé contenu dans un litre de lait entier est donc égal à $1.000-\frac{\mathrm{R}}{0,915}\left(\mathrm{~cm}^{3}\right)$.

On a done :

$$
\mathrm{K}_{1}=\frac{\mathrm{K}}{1.000-\frac{\mathrm{R}}{0,915}} \times 1.000=\frac{\mathrm{K}}{1-\frac{\mathrm{R}}{915}}
$$

et, inversement : $K=K_{1}\left(1-\frac{R}{915}\right)$.

Application pratique.

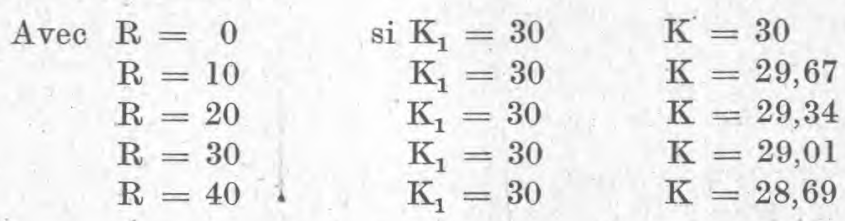

Ainsi, pour un lait donné de composition non grasse constante, 
le coefficient réel (que l'on admettait comme constant) diminue de 0,32 quand on passe d'un lait à 30 grammes à un lait ̀े 40 grammes par exemple.

La variation du coefficient est faible, certes. Elle ne sera pas considérée comme telle le jour où ce facteur interviendra dans la fixation du prix d'achat du lait...

D'autre part, certains écarts constatés expérimentalement dans l'étude pratique du coefficient ont sûrement leur origine dans cette variation de $\mathrm{K}$ en fonction de la richesse du lait. Nous proposons donc l'adoption du coefficient rectifié $\left(\mathrm{K}_{1}\right)$ rapporté au lait écrémé.

\section{Erreur due au sel}

Le salage de fabrication introduit une nouvelle cause d'erreur très importante dans le cas des pâtes molles. On compte en effet comme matière sèche du fromage (done venant du lait) le sel qui a été introduit et retenu en grande partie par certains fromages.

Dans le but de nous rendre compte de l'erreur ainsi commise, nous avons fait préparer parallèlement des camemberts salés et non salés. Nous en avons effectué l'analyse et dosé le sel dans les deux séries (les fromages non salés contiennent un peu de chlorure de sodium provenant du lait). Voici ce que nous avons constaté :

\begin{tabular}{|c|c|c|c|c|c|c|}
\hline \multirow{2}{*}{ r } & \multicolumn{2}{|c|}{ Camembert No 1} & \multicolumn{2}{|c|}{ Camembert $\mathrm{N}^{\circ} 2$} & \multicolumn{2}{|c|}{ Camembert $\mathrm{N}^{\circ} 3$} \\
\hline & Non salé & Salé & Non salé & Salé & Non salé & Salé \\
\hline Poids à l'analyse $\ldots \ldots \ldots$ & 285 & 307 & 300 & 292 & 300 & 303 \\
\hline $\mathrm{NaCl} \%$ de fromage frais. & 0,29 & 3,33 & 0,35 & 3,74 & 0,35 & 3,51 \\
\hline $\mathrm{NaCl} \%$ desec $\ldots . . \ldots$. & 1,03 & 10,56 & 1,26 & 11,93 & 1,25 & 11,03 \\
\hline NaCl total par fromage... & 0,82 & 10,22 & 1,05 & 10,92 & 1,05 & 10,63 \\
\hline $\begin{array}{l}\mathrm{NaCl} \text { "apporté " par fro- } \\
\text { mage } \ldots \ldots \ldots \ldots \ldots\end{array}$ & - & 9,40 & - & 9,87 & - & 9,58 \\
\hline $\begin{array}{l}\text { Matière sèche totale non } \\
\text { grasse } \ldots \ldots \ldots \ldots \ldots\end{array}$ & 69,74 & 87,43 & 72,98 & 83,05 & 70,80 & 85,87 \\
\hline $\begin{array}{r}\text { Matière sèche non grasse, } \\
\text { sel déduit } \ldots \ldots \ldots \ldots\end{array}$ & 69,74 & 78,03 & 72,98 & 73,18 & 70,80 & 76,29 \\
\hline Coefficient $\mathrm{K}$ non corrigé. & 28,4 & 35,6 & 29,7 & 33,9 & 28,9 & 35,0 \\
\hline Coefficient $\mathrm{K}$ sel déduit . & 28,4 & 31,8 & 29,7 & 29,8 & 28,9 & 31,1 \\
\hline $\begin{array}{l}\text { Ecart de coefficient dû au } \\
\text { sel } \ldots \ldots \ldots \ldots \ldots\end{array}$ & - & 3,8 & - & 4,1 & 一 & 3,9 \\
\hline
\end{tabular}

Nota. - Les fromages 1 et 3 non salés présentent un coefficient de rendement anormal (inférieur au coefficient rectifié des fromages salés alors qu'il devrait, théoriquement, lui être égal). Cela est 
sans importance puisque la détermination réelle se fera sur fromages salés, comme d'usage, avec correction due au sel dosé.

En négligeant l'influence du sel, on trouve donc des coefficients de rendement nettement supérieurs à la réalité. Les taux de sels retenus sont variables suivant les conditions de la fabrication d'un même type de fromages. (Ils le sont bien davantage quand on passe d'un type à l'autre.)

Si l'on veut caractériser le lait avec un peu de précision au point de vue de sa valeur fromagère, il est donc indispensable d'effectuer cette correction de sel. Le seul moyen d'y parvenir consiste à doser le sel total du fromage en adoptant un terme soustractif constant (à déterminer) pour le sel naturel du fromage. Si l'on négligeait ce terme soustractif, on commettrait, certes, une erreur, mais bien moins importante que celle qui consistait à considérer le sel de fabrication comme extrait sec dû au lait.

$$
\text { ** } *
$$

\section{En résumé.}

Nous proposons, dans le cadre de l'étude générale du coefficient de rendement en fromagerie et de ses variations :

10 D'expérimenter, pour le calcul de ce coefficient, des fabrications de laboratoire sur un ou deux fromages; ces fabrications en petit permettant une grande précision au point de vue des mesures et des analyses. (Les comparer à des essais semi-industriels.)

$2^{\circ}$ D'adopter une nouvelle définition du coefficient de rendement dans laquelle on déduira le sel (dosé) de l'extrait sec du fromage et on ramènera ce coefficient “sel déduit» à un litre de lait écrémé (à l'aide de la formule donnant $K_{1}$ en fonction de $K$ ). On obtiendra ainsi un "coefficient rectifé » qui se rapprochera davantage dés caractères d'une constante du lait utilisé.

Ces modifications nous paraissent indispensables au progrès de la question et à l'utilisation éventuelle de cette donnée dans le calcul du prix d'achat du lait destiné à la fromagerie. 